\title{
Face-Centered Anisotropic Surface Impedance Boundary Conditions in FDTD
}

\author{
Ian D. Flintoft ${ }^{\circledR}$, Senior Member, IEEE, Samuel A. Bourke, John F. Dawson ${ }^{\circledR}$, Member, IEEE, \\ Jesus Alvarez, Member, IEEE, Miguel Ruiz Cabello, Martin P. Robinson, Member, IEEE, \\ and Salvador G. Garcia ${ }^{\circledR}$, Senior Member, IEEE
}

\begin{abstract}
Thin-sheet models are essential to allow shielding effectiveness of composite enclosures and vehicles to be modeled. Thin dispersive sheets are often modeled using surface-impedance models in finite-difference time-domain (FDTD) codes in order to deal efficiently with the multiscale nature of the overall structure. Such boundary conditions must be applied to collocated tangential electric and magnetic fields on either side of the surface; this is usually done on the edges of the FDTD mesh cells at the electric field sampling points. However, these edge-based schemes are difficult to implement accurately on stair-cased surfaces. Here, we present a novel face-centered approach to the collocation of the fields for the application of the boundary condition. This approach naturally deals with the ambiguities in the surface normal that arise at the edges on stair-cased surfaces, allowing a simpler implementation. The accuracy of the new scheme is compared to edge-based and conformal approaches using both planar sheet and spherical shell canonical test cases. Staircasing effects are quantified and the new face-centered scheme is shown have up to 3-dB lower error than the edge-based approach in the cases considered, without the complexity and computational cost of conformal techniques.
\end{abstract}

Index Terms-Finite-difference time domain (FDTD), impedance network boundary condition, surface-impedance boundary condition (SIBC).

\section{INTRODUCTION}

$\mathbf{S}$ URFACE-IMPEDANCE boundary conditions (SIBCs) are one approach to improving the efficiency of multiscale electromagnetic simulations required for many applications

Manuscript received July 24, 2017; revised October 1, 2017 and November 2, 2017; accepted November 11, 2017. Date of publication December 11, 2017; date of current version February 5, 2018. This work was supported by the U.K. Engineering and Physical Sciences Research Council through the Flapless Air Vehicle Integrated Industrial Research Programme under Grant GR/S71552/01, in part by the European Community's Seventh Framework Programme under Grant FP7/2007-2013, in part by the High Intensity Radiated Field Synthetic Environment Research Project under Grant 205294, in part by the Spanish MINECO, EU FEDER under Project TEC2013-48414-C3-01 and Project TEC2016-79214-C3-3-R, and in part by J. de Andalucia, Spain under Project P12-TIC-1442. This paper is an expanded version from the IEEE MTT-S International Symposium Conference on Numerical Electromagnetic Modeling and Optimization for RF, Microwave and Teraherz Applications, May 17-19, 2017, Seville, Spain. (Corresponding author: John F. Dawson.)

I. D. Flintoft, S. A. Bourke, J. F. Dawson, and M. P. Robinson are with the Department of Electronic Engineering, University of York, York, YO10 5DD, U.K. (e-mail: ian.flintoft@york.ac.uk; samuel.bourke@ york.ac.uk; john.dawson@york.ac.uk; martin.robinson@york.ac.uk).

J. Alvarez is with Airbus Defence and Space, 28906 Getafe, Spain (e-mail: jesus@ieee.org).

M. R. Cabello and S. G. Garcia are with the Department of Electromagnetism and Matter Physics, University of Granada, 18071 Granada, Spain (e-mail: mcabello@ugr.es; salva@ugr.es).

Color versions of one or more of the figures in this paper are available online at http://ieeexplore.ieee.org.

Digital Object Identifier 10.1109/TMTT.2017.2778059 in all frequency ranges such as full aircraft electromagnetic response assessment [1], [2] and microwave [3] and plasmonic [4] devices. They form the basis for modeling many types of thin material surfaces, such as carbon fiber composites [5], composites with protective wire meshes [6], or graphene [4]. The efficiency is achieved by removing the material's internal structure from the computational space and replacing it with a behavioral model of the tangential electromagnetic fields on either side of the surface. This behavioral model takes the form of a frequency-dependent impedance matrix relating the transverse electric (TE) and transverse magnetic (TM) fields. It is in essence, a two-sided generalization of the one-sided SIBC used to model a metallic boundary with finite conductivity [7].

Since the development of the FDTD method, a wide range of methods have been applied to represent and implement the frequency-dependent surface impedance matrix in FDTD simulations; a short review of recent developments can be found in [9]. The most common approaches use partial fraction representations of the impedance matrix elements, typically implemented using recursive convolution algorithms in the discrete FDTD mesh, and enforce the SIBC on the mesh edges [5]. Other approaches such as equivalent circuits [10] or subgridding [11], [12] have also been investigated. Careful treatment of the intersection of SIBC faces is necessary in order to resolve the inherent ambiguity of the surface normal on stair-cased edges when curved surfaces are approximated on a structured mesh. Conformal algorithms have been developed [13]-[15]; however, these techniques are more computationally expensive and a tradeoff between efficiency and accuracy must be considered for their particular application.

In this paper, we present a detailed explanation of a novel face-centered approach to implementing a two-sided SIBC in FDTD first described in [16], and present additional results showing the performance of the method. An implementation of the method is available in the University of York, Vulture code. The approach adopted removes the ambiguity in the orientation of the surface normal on SIBC surface edges and corners where the edge-based approach has to be applied [5]. While much recent work has focused on planar sheet material validation cases for thin-sheet boundaries, we also investigate the behavior of the SIBC for more realistic curved surfaces. For the time integration technique, we employ cascades of second-order digital filters, which have previously been applied within the transmission-line matrix method [17]. 


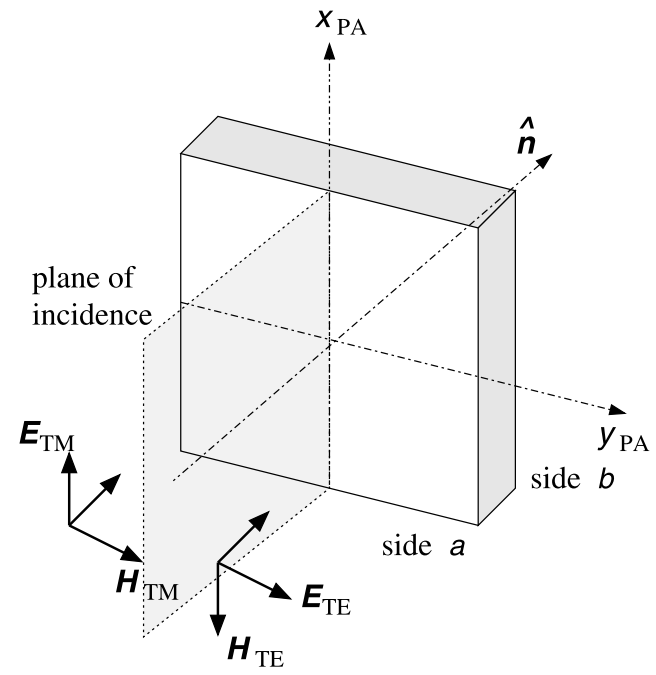

Fig. 1. Reflection and transmission of TM and TE polarized waves from a plane boundary in the principal axis coordinate system of the material's impedance matrix.

These cascaded filters offer more flexibility in optimizing the numerical performance of the discrete time algorithm than the widely used recursive convolution algorithm, which is analogous to a parallel first-order filter implementation and can limit the dynamic range and accuracy of the results due to the finite arithmetic precision.

The SIBC concept and nomenclature is introduced in Section II and the digital filter implementation of its dispersive matrix elements is given in Section III. The facecentered FDTD scheme is detailed in Section IV and the overall approached validated using two canonical test cases in Section V. We draw conclusions about the approach in Section VI.

\section{Definition And Alignment of SiBCs}

A two-sided SIBC relates the tangential electric and magnetic fields on either side of a thin sheet of material using an impedance matrix. For an anisotropic material, the tangential fields are first resolved into TE and TM components, as shown in Fig. 1. A natural convention for defining the surface impedance matrix $\bar{Z}(\omega)$ is with the TM and TE transverse fields blocked together on either side of the boundary, so that

$$
\left[\begin{array}{l}
E_{\mathrm{TM}}^{a} \\
E_{\mathrm{TM}}^{b} \\
E_{\mathrm{TE}}^{a} \\
E_{\mathrm{TE}}^{b}
\end{array}\right]=\bar{Z}(\omega)\left[\begin{array}{c}
H_{\mathrm{TM}}^{a} \\
-H_{\mathrm{TM}}^{b} \\
H_{\mathrm{TE}}^{a} \\
-H_{\mathrm{TE}}^{b}
\end{array}\right]
$$

where " $a$ " and " $b$ " denote the two sides of the boundary.

The TM and TE polarizations are defined with reference to the local principal axis reference system on the surface of the material. If we consider a $z$-normal boundary then the SIBC in the global coordinate system can be written

$$
\left[\begin{array}{c}
E_{x}^{a} \\
E_{x}^{b} \\
E_{y}^{a} \\
E_{y}^{b}
\end{array}\right]=\bar{B}(\alpha) \bar{Z}(\omega) \bar{A}(\alpha)\left[\begin{array}{c}
H_{x}^{a} \\
H_{x}^{b} \\
H_{y}^{a} \\
H_{y}^{b}
\end{array}\right]
$$

where $\bar{A}(\alpha)$ and $\bar{B}(\alpha)$ are rotation matrices that are functions of the angle $\alpha$ between the principal axes of the material and $x$-axis of the mesh.

\section{Digital Filter Representation OF THE SIBC FREQUENCY RESPONSE}

The SIBC is typically determined from a theoretical model [18], another high-resolution simulation or from measurements [19]. The impedance matrix data are then fitted to a partial fraction expansion (PFE) of the form

$$
Z_{p q}(s)=Z_{p q}^{\infty}+\sum_{m=1}^{N_{p q}} \frac{r_{p q}^{m}}{s-p_{p q}^{m}}
$$

where $s=j \omega$ is the Laplace variable, $r_{p q}^{m}$ and $p_{p q}^{m}$ are $m=1, \ldots, N_{p q}$ residues and poles (real or complex-conjugate pairs), and $Z_{p q}^{\infty}$ are the asymptotic high frequency responses. A number of approaches to fitting the PFE can be used, including deterministic fitting with a "vector fit" algorithm [20] and stochastic optimization using a genetic algorithm.

However, the PFE is generated, it is essential to ensure that it defines a stable, passive, and causal system over the entire frequency range of the simulation. Causality is automatically fulfilled by the PFE in (3), for which Kramers-Kronig relationships hold, and stability can be easily enforced by requiring $\operatorname{Re}\left[p_{p q}^{m}\right] \leq 0$. The passivity constraint is more difficult to enforce in general and may require an iterative procedure to be applied in the PFE fitting.

The SIBC response must be implemented as a discretetime algorithm. One approach to achieving this is to use a recursive convolution algorithm to directly evaluate the convolutions [5], [21]. Here, we use a digital filter realization of the impedance matrix elements. A number of well-known methods are available for mapping the $s$ plane PFE onto a discrete $z$ plane transfer function, including the impulse invariant $z$-transform, the matched $z$-transform, and the bilinear $z$-transform (BZT) [22]. Here, we chose the BZT as it is less susceptible to high frequency aliasing effects.

Whichever transform is used the resulting $z$ plane poles and zeros are partitioned into $\left\lceil N_{p q} / 2\right\rceil$ pairs to form a cascade of second-order-sections (SOSs) for each matrix element

$$
Z_{p q}(z)=g_{p q} \prod_{m=1}^{\left\lceil N_{p q} / 2\right\rceil} \frac{\left(\beta_{p q}^{0 ; m}+\beta_{p q}^{1 ; m} z^{-1}+\beta_{p q}^{2 ; m} z^{-2}\right)}{\left(1+\alpha_{p q}^{1 ; m} z^{-1}+\alpha_{p q}^{2 ; m} z^{-2}\right)}
$$

where the coefficients of the $m$ th section are denoted $\alpha_{p q}^{u ; m}$ and $\beta_{p q}^{u ; m}$ and the overall gain $g_{p q}$ is determined by matching the low-frequency response to the $s$ plane PFE. The ordering of the poles and zeros can be used to optimize the dynamic range and rounding error of the numerical approximation [22]. Each of the SOSs can be efficiently implemented as a direct-form-II-transpose filter, as shown in Fig. 2.

\section{EXPlicit FACE-CENTEREd FDTD AlgORITHM}

We construct an FDTD scheme on a uniform primary mesh defined by the nodes $(x, y, z)=(i \Delta x, j \Delta y, k \Delta z)$ with 


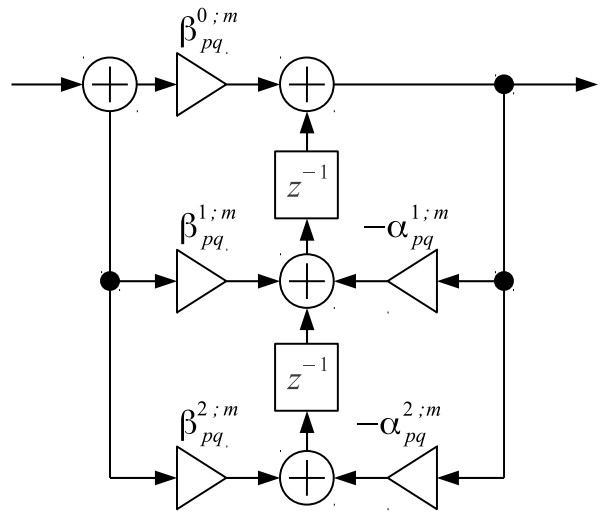

Fig. 2. The $m$ th direct-form-II-transpose second-order filter section of the SOS cascade for impedance matrix element $Z_{p q}$.

the electric fields centered on the primary mesh edges at integer time steps $t=n \Delta t$ and the magnetic fields normal to the corresponding faces at half-integer time steps [23]. Here, $\Delta x, \Delta y$, and $\Delta z$ are the mesh sizes in the respective directions and $\Delta t$ is the time step; fields on the mesh, $\psi(i \Delta x, j \Delta y, k \Delta z ; n \Delta t)$, are denoted compactly as $\psi_{i, j, k}^{n}$. An explicit update scheme is adopted for linking the SIBC algorithm into an FDTD code [5], [24]. The SIBC must be enforced at the same positions and times; here, we impose the SIBC at the face centers of the primary mesh at the electric field sampling times using spatial interpolation algorithms. This makes the treatment of intersecting SIBC faces very simple, at the expense of some extra averaging.

Since the tangential electric fields lie in the plane of the SIBC boundary, the values to either side of the boundary must be stored separately on every face on which the SIBC is imposed. Each part of the algorithm is discussed in detail in the following sections.

\section{A. Electric Field Updates}

The electric fields throughout the mesh are first updated using the standard FDTD equations [24]. It is then advantageous to set the tangential electric fields on the mesh at the SIBC edges to zero. In this face-centered approach, we choose to store the actual tangential electric fields at the face centers in separate storage associated with each SIBC face. The SIBC for a $z$-normal face centered on $(i+1 / 2, j+1 / 2, k)$ is given by

$$
\left[\begin{array}{c}
E_{x}^{a} \\
E_{x}^{b} \\
E_{y}^{a} \\
E_{y}^{b}
\end{array}\right]_{i+\frac{1}{2}, j+\frac{1}{2}, k}^{n}=\bar{B}(\alpha) Z_{a, b}^{\mathrm{TM}, \mathrm{TE}} \otimes \bar{A}(\alpha)\left[\begin{array}{c}
H_{x}^{a} \\
H_{x}^{b} \\
H_{y}^{a} \\
H_{y}^{b}
\end{array}\right]_{i+\frac{1}{2}, j+\frac{1}{2}, k}^{n}
$$

where $Z_{a, b}^{\mathrm{TM}, \mathrm{TE}}$ is the impulse response of the impedance matrix and $\otimes$ denotes convolution. The main difficulty is therefore the determination of the tangential magnetic fields at the correct sampling locations and times. The spatial interpolation scheme shown in Fig. 3 is first applied to allow the determination

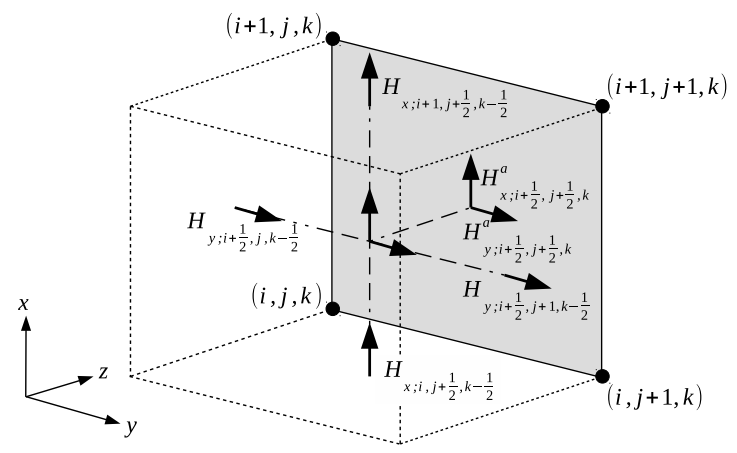

Fig. 3. Spatial interpolation of the tangential magnetic fields to the SIBC face centers for the electric field update on the $z$-low side of the $z$-normal face centered on $(i+1 / 2, j+1 / 2, k)$. The SIBC face is shaded.

of the tangential components on the central normal axis of the face, half a cell either side of the boundary, at time $t=(n-1 / 2) \Delta t$

$$
\begin{aligned}
H_{x ; i+\frac{1}{2}, j+\frac{1}{2}, k-\frac{1}{2}}^{n-\frac{1}{2}} & =\frac{1}{2}\left[H_{x ; i, j+\frac{1}{2}, k-\frac{1}{2}}^{n-\frac{1}{2}}+H_{x ; i+1, j+\frac{1}{2}, k-\frac{1}{2}}^{n-\frac{1}{2}}\right] \\
H_{x ; i+\frac{1}{2}, j+\frac{1}{2}, k+\frac{1}{2}}^{n-\frac{1}{2}} & =\frac{1}{2}\left[H_{x ; i, j+\frac{1}{2}, k+\frac{1}{2}}^{n-\frac{1}{2}}+H_{x ; i+1, j+\frac{1}{2}, k+\frac{1}{2}}^{n-\frac{1}{2}}\right] \\
H_{y ; i+\frac{1}{2}, j+\frac{1}{2}, k-\frac{1}{2}}^{n-\frac{1}{2}} & =\frac{1}{2}\left[H_{y ; i+\frac{1}{2}, j, k-\frac{1}{2}}^{n-\frac{1}{2}}+H_{y ; i+\frac{1}{2}, j+1, k-\frac{1}{2}}^{n-\frac{1}{2}}\right] \\
H_{y ; i+\frac{1}{2}, j+\frac{1}{2}, k+\frac{1}{2}}^{n-\frac{1}{2}} & =\frac{1}{2}\left[H_{y ; i+\frac{1}{2}, j, k+\frac{1}{2}}^{n-\frac{1}{2}}+H_{y ; i+\frac{1}{2}, j+1, k+\frac{1}{2}}^{n-\frac{1}{2}}\right] .
\end{aligned}
$$

Then, to attain spatial and temporal co-location with the electric field at the face center, the magnetic fields on the boundary at $t=n \Delta t$ are estimated as [5]

$$
\begin{aligned}
& H_{x ; i+\frac{1}{2}, j+\frac{1}{2}, k}^{a ; n} \approx H_{x ; i+\frac{1}{2}, j+\frac{1}{2}, k-\frac{1}{2}}^{n-\frac{1}{2}} \\
& H_{x ; i+\frac{1}{2}, j+\frac{1}{2}, k}^{b ; n} \approx H_{x ; i+\frac{1}{2}, j+\frac{1}{2}, k+\frac{1}{2}}^{n-\frac{1}{2}} \\
& H_{y ; i+\frac{1}{2}, j+\frac{1}{2}, k}^{a ; n} \approx H_{y ; i+\frac{1}{2}, j+\frac{1}{2}, k-\frac{1}{2}}^{n-\frac{1}{2}} \\
& H_{y ; i+\frac{1}{2}, j+\frac{1}{2}, k}^{b ; n} \approx H_{y ; i+\frac{1}{2}, j+\frac{1}{2}, k+\frac{1}{2}}^{n-\frac{1}{2}} .
\end{aligned}
$$

This low-order approximation limits the overall accuracy of the algorithm and has been suggested as a possible source of the long term instability sometimes observed in SIBC implementations in FDTD [24]. Recently, a more accurate approximation derived from the field equations has been developed, treating the PFE matrix elements of the boundary in a similar way to multipole Debye materials [9]. The authors demonstrate that their method is long term stable for some typical materials in pseudo-1-D waveguide models with planar materials in the cross section, though no formal proof of stability is given. However, the method is incompatible with the SOS cascade filter implementation of the impedance matrix elements, and since our main interest here is in the face-centered approach, we apply the simple approximation in (7) and take the input to the SIBC filters, described 
in Section III, to be

$$
\begin{aligned}
& {\left[\begin{array}{l}
H_{1} \\
H_{2} \\
H_{3} \\
H_{4}
\end{array}\right]_{i+\frac{1}{2}, j+\frac{1}{2}, k}^{n}\left[\begin{array}{c}
H_{x ; i, j+\frac{1}{2}, k-\frac{1}{2}}^{n-\frac{1}{2}}+H_{x ; i+1, j+\frac{1}{2}, k-\frac{1}{2}}^{n-\frac{1}{2}} \\
H_{x ; i, j+\frac{1}{2}, k+\frac{1}{2}}^{n-\frac{1}{2}}+H_{x ; i+1, j+\frac{1}{2}, k+\frac{1}{2}}^{n-\frac{1}{2}} \\
H_{y ; i+\frac{1}{2}, j, k-\frac{1}{2}}^{n-\frac{1}{2}}+H_{y ; i+\frac{1}{2}, j+1, k-\frac{1}{2}}^{n-\frac{1}{2}} \\
H_{y ; i+\frac{1}{2}, j, k+\frac{1}{2}}^{n-\frac{1}{2}}+H_{y ; i+\frac{1}{2}, j+1, k+\frac{1}{2}}^{n-\frac{1}{2}}
\end{array}\right] .}
\end{aligned}
$$

The output of the filters $\left[\begin{array}{llll}E_{1} & E_{2} & E_{3} & E_{4}\end{array}\right]_{i+(1 / 2), j+(1 / 2), k}^{n}$ is then transformed into the updated tangential electric fields using

$$
\left[\begin{array}{c}
E_{x}^{a} \\
E_{x}^{b} \\
E_{y}^{a} \\
E_{y}^{b}
\end{array}\right]_{i+\frac{1}{2}, j+\frac{1}{2}, k}^{n}=\bar{B}(\alpha)\left[\begin{array}{c}
E_{1} \\
E_{2} \\
E_{3} \\
E_{4}
\end{array}\right]_{i+\frac{1}{2}, j+\frac{1}{2}, k}^{n}
$$

\section{B. Magnetic Field Updates}

The standard magnetic field update equations are first applied across the entire mesh. For components normal to SIBC faces these will use the zeroed values of the tangential electric field edges stored in the mesh, thereby automatically zeroing the normal magnetic field. Corrections are then applied using the actual tangential electric fields on the SIBC. Consider, for example, the update of $H_{x}$ near a $z$-normal SIBC, as shown in Fig. 4. The standard update

$$
\begin{aligned}
H_{x ; i, j+\frac{1}{2}, k+\frac{1}{2}}^{n+\frac{1}{2}}=H_{x ; i, j+\frac{1}{2}, k+\frac{1}{2}}^{n-\frac{1}{2}}+C_{i, j+\frac{1}{2}, k+\frac{1}{2}}^{h x e} & \\
\times & {\left[\begin{array}{c}
\frac{1}{\Delta z_{k}}\left\{E_{y ; i, j+\frac{1}{2}, k+1}^{n}-E_{y ; i, j+\frac{1}{2}, k}^{b ; n}\right\} \\
+\frac{1}{\Delta y_{j}}\left\{E_{z ; i, j, k+\frac{1}{2}}^{n}-E_{z ; i, j+1, k+\frac{1}{2}}^{n}\right\}
\end{array}\right] }
\end{aligned}
$$

is first applied with $E_{y ; i, j+(1 / 2), k}^{b ; n}=0$; this term must therefore be applied as a correction. Here, $C_{i, j+(1 / 2), k+(1 / 2)}^{h x e}$ is the standard magnetic field update coefficient [23]. Rather than apply the SIBC corrections on an edge-by-edge basis it is more consistent with the face-centered approach to split the corrections amongst the faces. For example, the correction for tangential magnetic field update in (10) is applied in two parts

$$
\begin{aligned}
H_{x ; i, j+\frac{1}{2}, k+\frac{1}{2}}^{n+\frac{1}{2}} & H_{x ; i, j+\frac{1}{2}, k+\frac{1}{2}}^{n-\frac{1}{2}}-\frac{C_{i, j+\frac{1}{2}, k+\frac{1}{2}}^{h x e}}{2 \Delta z} E_{y ; i+\frac{1}{2}, j+\frac{1}{2}, k}^{b ; n} \\
H_{x ; i+1, j+\frac{1}{2}, k+\frac{1}{2}}^{n+\frac{1}{2}}= & H_{x ; i+1, j+\frac{1}{2}, k+\frac{1}{2}}^{n-\frac{1}{2}} \\
& -\frac{C_{i+1, j+\frac{1}{2}, k+\frac{1}{2}}^{h x e}}{2 \Delta z} E_{y ; i+\frac{1}{2}, j+\frac{1}{2}, k}^{b ; n}
\end{aligned}
$$

with each face responsible for applying its part of the correction. The electric fields on the SIBC face edges are estimated from the average of those at the neighboring face centers,

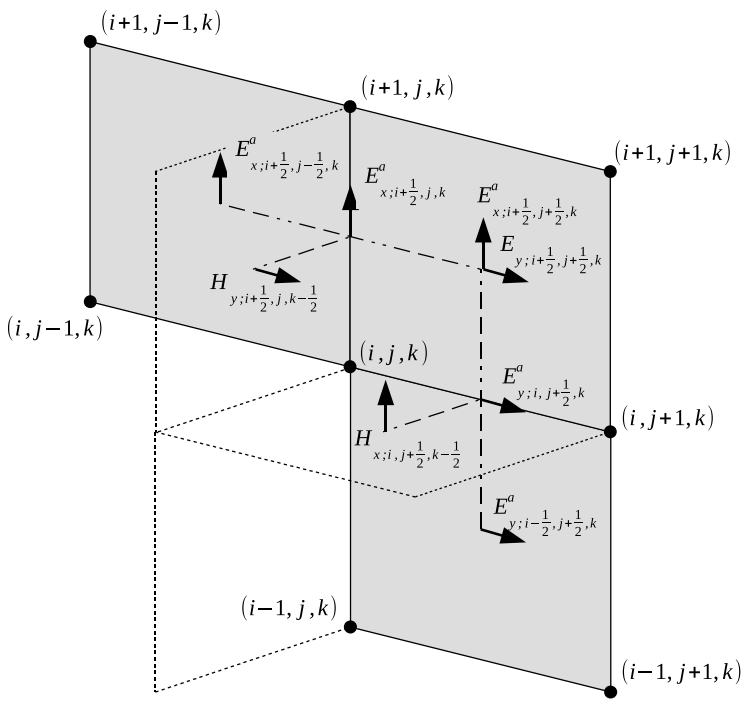

Fig. 4. Spatial interpolation of the tangential electric fields on the SIBC faces (shaded) for the tangential magnetic field updates adjacent to the SIBC on the $z$-low side of a $z$-normal face.

which are in turn determined by the updated tangential electric fields from the SIBC filter output (9) by using a simple two-point average as illustrated in Fig. 4

$$
\begin{aligned}
& E_{y ; i, j+\frac{1}{2}, k}^{a ; n}=\frac{1}{2}\left[E_{y ; i-\frac{1}{2}, j+\frac{1}{2}, k}^{a ; n}+E_{y ; i+\frac{1}{2}, j+\frac{1}{2}, k}^{a ; n}\right] \\
& E_{y ; i, j+\frac{1}{2}, k}^{b ; n}=\frac{1}{2}\left[E_{y ; i-\frac{1}{2}, j+\frac{1}{2}, k}^{b ; n}+E_{y ; i+\frac{1}{2}, j+\frac{1}{2}, k}^{b ; n}\right] .
\end{aligned}
$$

Note that since the SIBC is applied as a correction to the required faces after the standard update equations, it has little impact on the parallelization performance of the overall code.

\section{Treatment of Edges and Corners}

In the edge-centered formulation the treatment of the edges and corners at the intersection of thin boundaries is critical to the accuracy and stability of the code. The problem is essentially caused by the ambiguity of the normal vector of the surface at edges and corners of boundaries and can be exacerbated by the nature of the staggered FDTD mesh and also the polarization requirements for anisotropic boundaries. However, in the face-based approach to implementing the SIBC the tangential fields at the face centers are always well defined and unambiguous.

Two potential problems remain one for the electric field update and the other for the magnetic field update. Regarding the electric field in the SIBC updates near a corner, one of the tangential magnetic fields in the average used to determine the tangential field at the face center will be a normal field on the intersecting surface, as depicted in Fig. 5(a). The normal fields on the mesh are null, so in this case the average is set to the value of the other tangential field component so the update equation becomes

$$
H_{y ; i+\frac{1}{2}, j+\frac{1}{2}, k}^{a}=\frac{1+\operatorname{adj}}{2}\left[H_{y ; i+\frac{1}{2}, j, k-\frac{1}{2}}^{b}+H_{y ; i+\frac{1}{2}, j+1, k-\frac{1}{2}}^{b}\right]
$$




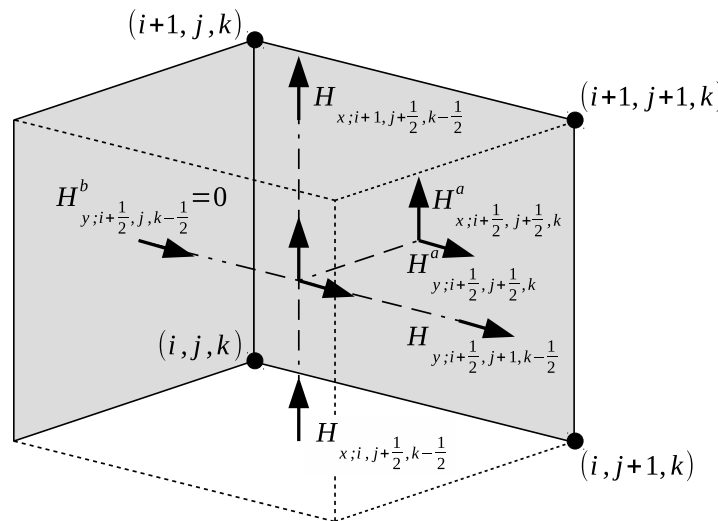

(a)

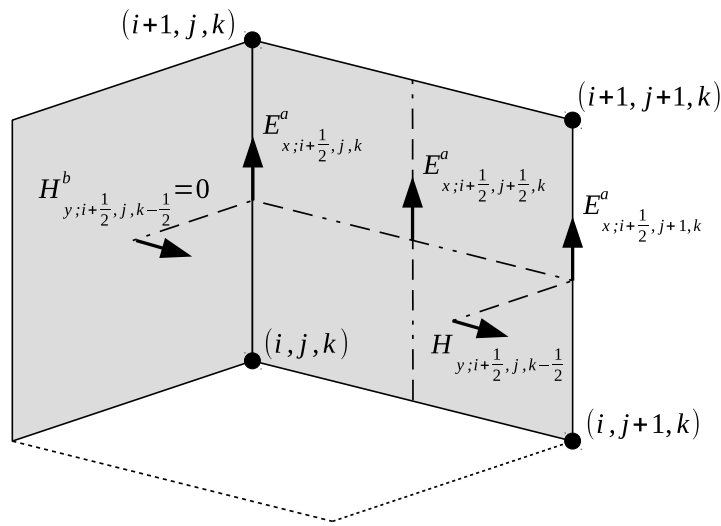

(b)

Fig. 5. Intersection between two SIBC faces (shaded) in the face centric approach showing the fields involved in (a) tangential magnetic field update adjacent to the SIBC and (b) tangential electric field update on the SIBC.

where $\operatorname{adj}=1$ if at a corner and zero elsewhere. This approximation appears to be reasonable given that the stair-cased surface is only an approximation to the true 3-D surface of the physical object.

Now consider the problem arising in the update of the tangential magnetic fields adjacent to the boundary as shown in Fig. 5(b). Near a corner, the magnetic field update will average together tangential electric fields that are on opposite sides of the intersecting boundary, a potentially disastrous occurrence. However, all such tangential magnetic field components are also a normal component of the intersecting boundary and are therefore null, as they are zeroed by the circulating electric field edges in the boundary. The face-based approach to implementing the SIBC thus naturally handles the intersection of SIBC boundaries with no modification.

\section{RESULTS}

Basic validation of the face-centered SIBC implementation has been achieved using the canonical test case of a planar sheet of material in the cross section of a parallel waveguide. The planar sheet is illuminated by a transverse electromagnetic wave excited by a partial total-field scattered-field boundary on one side and the electric field transmitted through the material is observed on the other side of the sheet. The shielding effectiveness (SE) of the material is determined as the ratio of the transmitted to

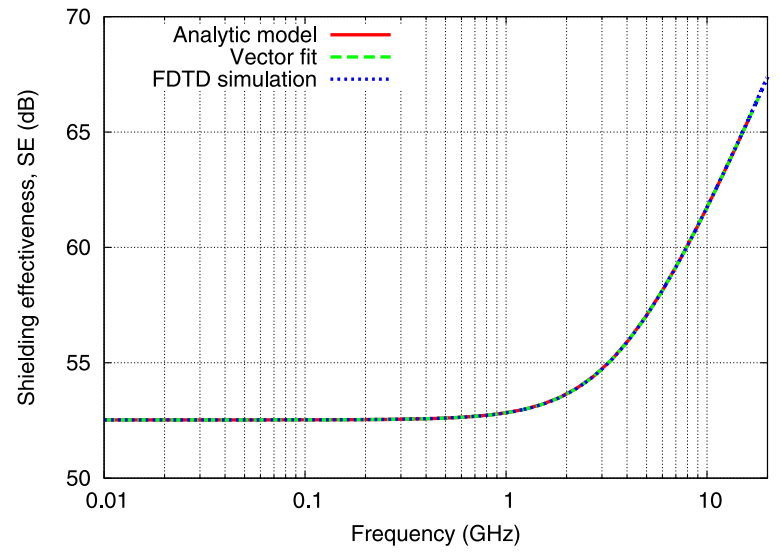

Fig. 6. SE of an isotropic metalized fabric.

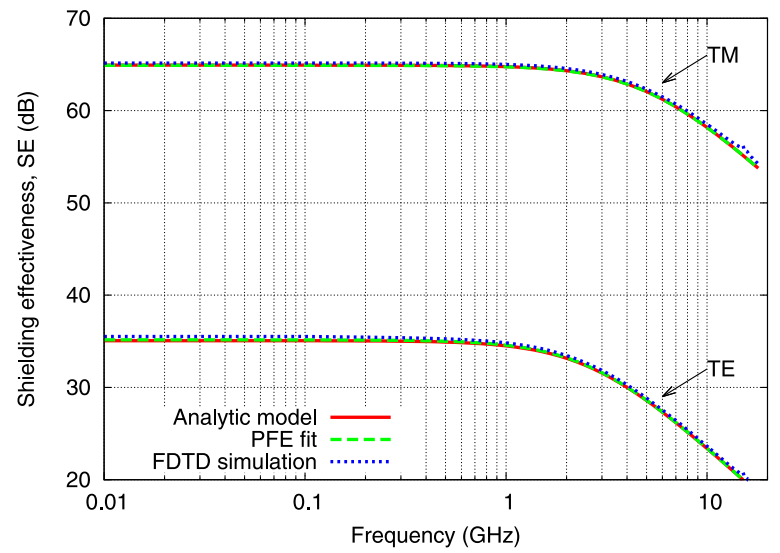

Fig. 7. SE of an anisotropic wire mesh.

incident electric field strength. The mesh size for the results presented below is $1 \mathrm{~mm}$. About 1.8 million iterations were required to achieve a good convergence due to the decay time of the impulse response of the impedance matrix.

The results for the first test case are shown in Fig. 6. The material consists of three layers-a $60-\mu$ m-thick central lossless dielectric layer with a relative permittivity 2 sandwiched between two 1.6- $\mu \mathrm{m}$-thick metallic layers with conductivity $0.7 \mathrm{MS} / \mathrm{m}$. The impedance matrix of the laminate was determined using a cascaded transmission line model [18] and fitted to fourth-order PFEs using a vector fit algorithm [20]. This material has an isotropic-impedance matrix. Fig. 6 shows that the analytic input model, the $s$ plane PFE approximation, and the FDTD face-centered SIBC simulation result agree within a fraction of a decibel, demonstrating the accuracy of the digital filters and averaging algorithms.

Another example is shown in Fig. 7. This material is a model for a woven stainless steel wire mesh which has an anisotropic impedance matrix; an analytic model was again used to estimate this impedance matrix [6]. The difference in SE between the TE and TM polarizations is about $25 \mathrm{~dB}$. The agreement between analytic model, the PFE fit (sixth order in this case), and the FDTD face-centered SIBC simulation is again excellent. The principal axes of the material can easily be orientated in any direction on a cell-by-cell basis using the implemented algorithm. 


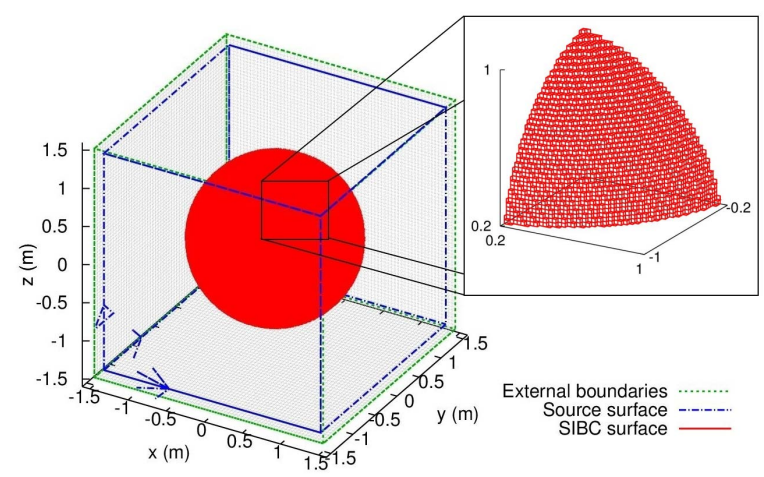

Fig. 8. Geometry of the hollow spherical shell test case. The shell radius is $1 \mathrm{~m}$ and it is illuminated by a $y$-polarized plane wave propagating along the $x$-direction generated by a total-field scattered field boundary.

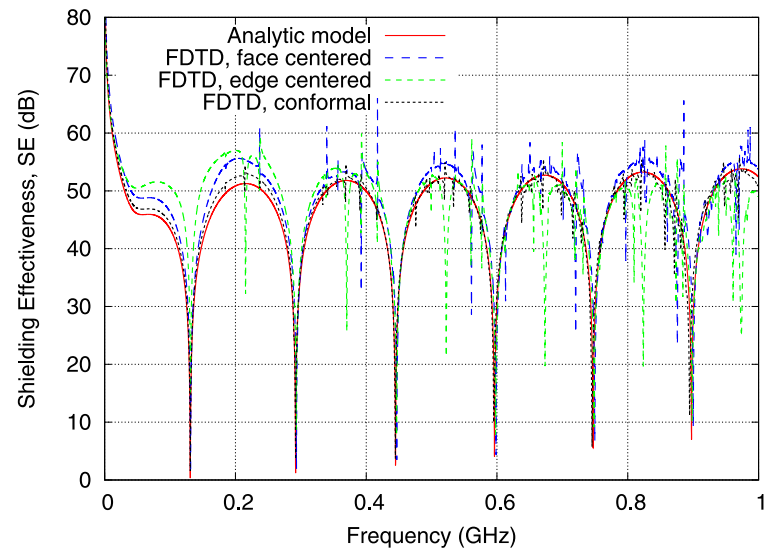

Fig. 9. SE at the center of the hollow spherical shell with $\sigma=1 \mathrm{kS} / \mathrm{m}$ and thickness $h=1 \mathrm{~mm}$ comparing the analytic solution to the different FDTD methods.

Fig. 8 shows a more complicated test case consisting of a 1-m-radius hollow spherical shell made from a simple conductive material. As the entire structure is curved, this case relies heavily on staircase approximations, making it ideal for measuring the limits of SIBCs on structured meshes. A uniform plane-wave was used to illuminate the sphere and the SE at the center of the shell was determined. The mesh size used in these FDTD models was $20 \mathrm{~mm}$. The SE values were stable within $\pm 0.05 \mathrm{~dB}$ (converged) for mesh sizes below $40 \mathrm{~mm}$. Using 600000 iterations $(20 \mu \mathrm{s})$ ensured good convergence in the time series. The problem took $0.326 \mathrm{~s}$ per iteration on an Intel(R) Core(TM) i7-4790 CPU. Due to the offset fields used by FDTD the electric field vector was determined by linear interpolation of immediately adjacent electric fields on the FDTD mesh. For validation, a relatively simple closed form result is available for the electric field at the shell center [25].

The results for the first spherical test case are shown in Fig. 9. In this case the shell material is a 1-mm-thick conductive sheet with a conductivity of $1 \mathrm{kS} / \mathrm{m}$. The new face-centered approach is compared to the usual edge-based approach implemented in the University of Granada FDTD code, and also to results found with a conformal SIBC approach, based on the combination of the SIBC technique

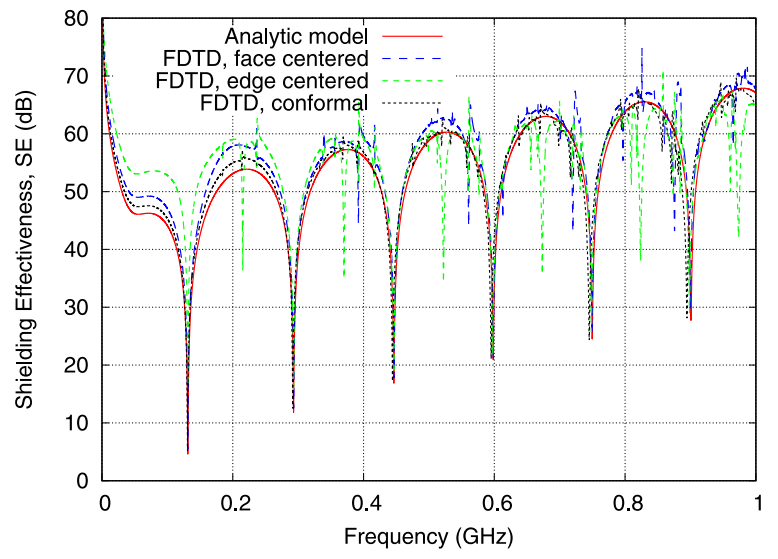

Fig. 10. SE at the center of the hollow spherical shell with $\sigma=200 \mathrm{~S} / \mathrm{m}$ and thickness $h=5 \mathrm{~mm}$ comparing the analytic solution to the different FDTD methods.

of [11] and the conformal method of [15]. The results for the edge-centered case show an error of about $6 \mathrm{~dB}$ around the first resonance, whereas the face-centered approach shows an error of about $3 \mathrm{~dB}$. This is an appreciable increase in accuracy, but there is still a noticeable difference in comparison to the analytic model which is fully attributed to stair-casing errors as demonstrated by the superior accuracy of the conformal results. Using feature selective validation (FSV) [26] the difference metrics $\left(\mathrm{ADM}_{\mathrm{tot}}\right.$ and $\left.\mathrm{FDM}_{\mathrm{tot}}\right)$ when compared to the analytic solution, were: 0.06 and 0.38 (excellent and good) for the conformal method; 0.13 and 0.61 (very good and fair) for the face-centered method; and 0.19 and 0.71 (very good and fair) for the edge-centered method.

A second spherical shell test case used a material with a conductivity of $200 \mathrm{~S} / \mathrm{m}$ and a thickness of $5 \mathrm{~mm}$. For these material parameters, the thickness of the shell varied from 1.4 to 4.4 "skin depths" between 100 and $1000 \mathrm{MHz}$. This is a wider range than for the first test case (which only spanned 0.6-2 "skin-depths") and means that at higher frequencies the SE should noticeably increase. Fig. 10 shows that this is indeed the case; again there is about $3-\mathrm{dB}$ error around the first resonance in the face-centered result and over 6-dB error in the edge-based result. The FSV ( $\mathrm{ADM}_{\mathrm{tot}}$ and $\left.\mathrm{FDM}_{\mathrm{tot}}\right)$ measures when compared to the analytic solution were: 0.09 and 0.4 (excellent and good) for the conformal method; 0.09 and 0.38 (excellent and good) for the face-centered method; and 0.2 and 0.55 (very good and fair) for the edgebased method. The face-centered approach gives better metrics than the conformal approach here as it seems to have a slightly better accuracy in the frequency of the minima which have a greater effect on the metrics than the larger errors in the magnitude near the maxima.

In both spherical shell test cases there was a noticeable reduction in spurious resonances immediately after the first real resonance in the face-centered results compared to the edge-based results, particularly in Fig. 10. This was due to a better representation of the physical geometry by the inherent corner treatment of the face-centered approach. Results found with the conformal technique further corroborate this claim. 


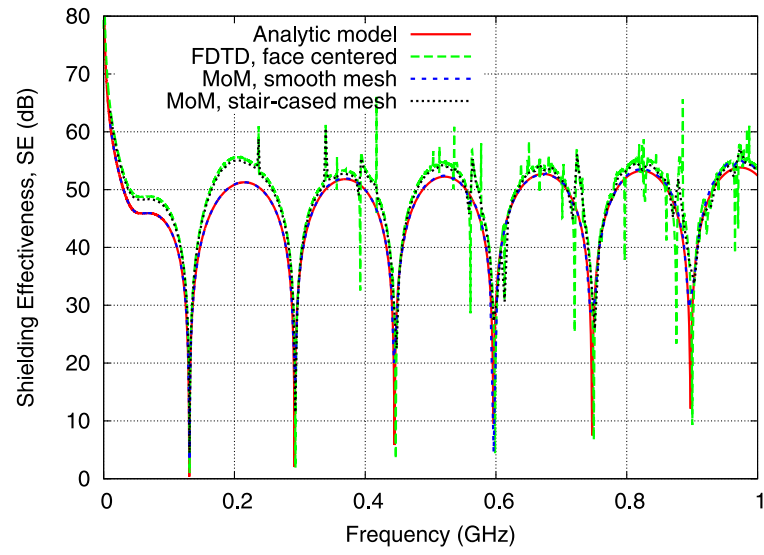

Fig. 11. SE of the hollow spherical shell with $\sigma=1 \mathrm{kS} / \mathrm{m}$ and thickness $h=1 \mathrm{~mm}$ showing MoM results with smooth and stair-cased meshes compared to the analytic model and face-centered FDTD results.

Further evidence that the remaining error in the facecentered scheme is predominantly due to stair-casing was obtained by modeling the spherical shell in the CONCEPT II method-of-moments (MoMs) code using both a highresolution smooth spherical surface mesh and an unstructured version of the stair-cased surface mesh (Fig. 8) used in the FDTD simulation. The size of the triangular elements in the unstructured MoM meshes was $50-100 \mathrm{~mm}$. These results are shown in Fig. 11. The MoM solution for the smooth mesh is almost identical to the analytic result while the MoM solution for the stair-cased mesh is very close to the facecentered FDTD simulation result. Even the first few sharp resonances that appear in the stair-cased FDTD result are replicated in the MoM solution. We have also replicated this in other commercial MoM and FDTD codes.

\section{CONCLUSION}

The surface impedance boundary approach is a computationally very efficient and flexible way to incorporate thin-sheet material properties in an FDTD simulation. It is applicable to any material for which it is possible to generate a passive and stable rational function impedance matrix model. The face-centered algorithm described here is particularly easy to implement since it automatically deals with most of the complexities associated with corners and interfaces between different surfaces. We have shown that this face-centered approach provides more accurate results than the edge-centered method when dealing with stair-cased geometries.

Ultimately, conformal techniques provide the best accuracy but we believe the techniques described here will be of interest to the modeling community due to the simplicity and efficiency of the implementation, along with improved accuracy compared to edge-based methods.

\section{ACKNOWLEDGMENT}

The authors would like to thank the Prof. M. Sarto and Dr. S. Greco from the Department of Astronautics, Electrical and Energetic Engineering, University of Rome Sapienza,
Rome, Italy, who supplied the analytic impedance model data for the planar material test cases in Section V.

\section{REFERENCES}

[1] M. Apra, M. D'Amore, K. Gigliotti, M. S. Sarto, and V. Volpi, "Lightning indirect effects certification of a transport aircraft by numerical simulation," IEEE Trans. Electromagn. Compat., vol. 50, no. 3, pp. 513-523, Aug. 2008.

[2] X. Meng, P. Sewell, S. Phang, A. Vukovic, and T. M. Benson, "Modeling curved carbon fiber composite (CFC) structures in the transmission-line modeling (TLM) method," IEEE Trans. Electromagn. Compat., vol. 57, no. 3, pp. 384-390, Jun. 2015.

[3] M. K. Karkkainen and P. M. T. Ikonen, "Finite-difference time-domain modeling of frequency selective surfaces using impedance sheet conditions," IEEE Trans. Antennas Propag., vol. 53, no. 9, pp. 2928-2937, Sep. 2005.

[4] V. Nayyeri, M. Soleimani, and O. M. Ramahi, "Modeling graphene in the finite-difference time-domain method using a surface boundary condition," IEEE Trans. Antennas Propag., vol. 61, no. 8, pp. 4176-4182, Aug. 2013.

[5] M. S. Sarto, "A new model for the FDTD analysis of the shielding performances of thin composite structures," IEEE Trans. Electromagn. Compat., vol. 41, no. 4, pp. 298-306, Nov. 1999.

[6] M. S. Sarto, S. Greco, and A. Tamburrano, "Shielding effectiveness of protective metallic wire meshes: EM modeling and validation," IEEE Trans. Electromagn. Compat., vol. 56, no. 3, pp. 615-621, Jun. 2014.

[7] J. G. Maloney and G. S. Smith, "The efficient modeling of thin material sheets in the finite-difference time-domain (FDTD) method," IEEE Trans. Antennas Propag., vol. 40, no. 3, pp. 323-330, Mar. 1992.

[8] K. Yee, "Numerical solution of initial boundary value problems involving Maxwell's equations in isotropic media," IEEE Trans. Antennas Propag., vol. AP-14, no. 3, pp. 302-307, May 1966.

[9] V. Nayyeri, M. Soleimani, and O. M. Ramahi, "A method to model thin conductive layers in the finite-difference time-domain method," IEEE Trans. Electromagn. Compat., vol. 56, no. 2, pp. 385-392, Apr. 2014.

[10] M. Feliziani, "Subcell FDTD modeling of field penetration through lossy shields," IEEE Trans. Electromagn. Compat., vol. 54, no. 2, pp. 299-307, Apr. 2012.

[11] M. R. Cabello et al., "A hybrid Crank-Nicolson FDTD subgridding boundary condition for lossy thin-layer modeling," IEEE Trans. Microw. Theory Techn., vol. 65, no. 5, pp. 1397-1406, May 2017.

[12] J. Chen, J. Li, and Q. H. Liu, "Designing graphene-based absorber by using HIE-FDTD method," IEEE Trans. Antennas Propag., vol. 65, no. 4, pp. 1896-1902, Apr. 2017.

[13] S. Dey and R. Mittra, "A locally conformal finite-difference time-domain (FDTD) algorithm for modeling three-dimensional perfectly conducting objects," IEEE Microw. Guided Wave Lett., vol. 7, no. 9, pp. 273-275, Sep. 1997.

[14] S. Benkler, N. Chavannes, and N. Kuster, "A new 3-D conformal PEC FDTD scheme with user-defined geometric precision and derived stability criterion," IEEE Trans. Antennas Propag., vol. 54, no. 6, pp. 1843-1849, Jun. 2006.

[15] M. R. Cabello, L. D. Angulo, J. Alvarez, A. R. Bretones, G. G. Gutierrez, and S. G. Garcia, "A new efficient and stable 3D conformal FDTD," IEEE Microw. Compon. Lett., vol. 26, no. 8, pp. 553-555, Aug. 2016.

[16] J. F. Dawson et al., "Face centered anisotropic surface impedance boundary conditions in FDTD: Improved performance of staircased mesh for shielding problems," in IEEE MTT-S Int. Microw. Symp. Dig., Seville, Spain, May 2017, pp. 260-262.

[17] J. A. Cole, J. F. Dawson, and S. J. Porter, "Efficient modelling of thin conducting sheets within the TLM method," in Proc. 3rd Int. Conf. Comput. Electromagn., Apr. 1996, pp. 45-50.

[18] C. L. Holloway, M. S. Sarto, and M. Johansson, "Analyzing carbonfiber composite materials with equivalent-Layer models," IEEE Trans. Electromagn. Compat., vol. 47, no. 4, pp. 833-844, Nov. 2005.

[19] I. D. Flintoft, J. F. Dawson, A. C. Marvin, and S. J. Porter, "Development of time-domain surface macro-models from material measurements," in Proc. 23rd Int. Rev. Prog. Appl. Comput. Electromagn., Verona, Italy, Mar. 2007, pp. 19-23.

[20] B. Gustavsen and A. Semlyen, "Rational approximation of frequency domain responses by vector fitting," IEEE Trans. Power Del., vol. 14, no. 3, pp. 1052-1061, Jul. 1999. 
[21] J. J. Akerson, "Finite difference time domain (FDTD) impedance boundary condition for thin finite conducting sheets," Prog. Electromagn. Res., vol. 31, pp. 1-30, 2001. [Online]. Available: http://www.jpier.org/PIER/pier.php?paper=0007011

[22] S. K. Mitra, Digital Signal Processing, 3rd ed. New York, NY, USA: McGraw-Hill, 2006.

[23] S. D. Gedney, "Introduction to the finite-difference time-domain (FDTD) method for electromagnetics," in Synthesis Lectures on Computational Electromagnetics, vol. 6, C. A. Balanis, Ed. San Rafael, CA, USA: Morgan Claypool, 2011, pp. 1-250.

[24] G. Kobidze, "Implementation of collocated surface impedance boundary conditions in FDTD," IEEE Trans. Antennas Propag., vol. 58, no. 7, pp. 2394-2403, Jul. 2010.

[25] M. Ansarizadeh, "Calculation of the shielding effectiveness of carbonfiber composite structures," M.S. thesis, Dept. Elect. Comput. Eng., Concordia Univ., Montréal, QC, Canada, 2013.

[26] IEEE Recommended Practice for Validation of Computational Electromagnetics Computer Modeling and Simulations, IEEE Standard 1597.2, 2010.

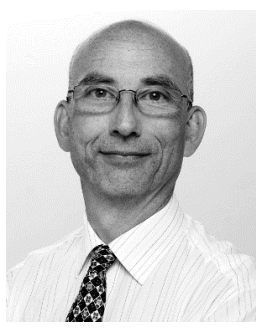

Ian D. Flintoft (M'00-SM'14) received the B.Sc. and Ph.D. degrees in physics from the University of Manchester, Manchester, U.K., in 1988 and 1994, respectively.

From 1996 to 2017, he was a Research Fellow with the Department of Electronic Engineering, University of York, York, U.K. He is currently a Senior Systems Engineer with Atkins, York. His current research interests include computational electromagnetics, model-based systems engineering, electromagnetic compatibility, and bioelectromagnetics.

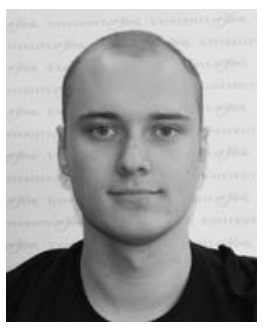

Samuel A. Bourke received the M.Phys. degree from the University of York, York, U.K., in 2015, where he is currently purusing the Ph.D. degree in electronics.

$\mathrm{He}$ is currently involved in the development of electromagnetic time domain solvers focusing on the finite-difference time-domain method and thin boundary models.

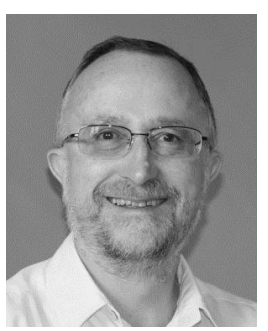

John F. Dawson (M'90) received the B.Sc. and D.Phil. degrees in electronics from the University of York, York, U.K., in 1982 and 1989, respectively.

$\mathrm{He}$ is currently a Senior Lecturer and the Deputy Leader of the Communication Technologies Research Group, University of York.

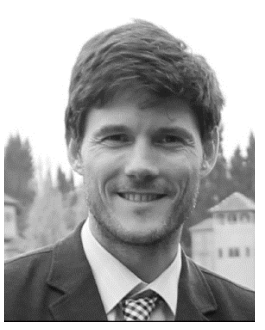

Jesus Alvarez (M'00) was born in Leon, Spain. $\mathrm{He}$ received the B.Sc. degree from the University of Cantabria, Santander, Spain, in 2001, the M.Sc. degree from the University Carlos III of Madrid, Getafe, Spain, in 2008, and the Ph.D. degree from the University of Granada, Granada, Spain, in 2013.

Since 2006, he has been an Antenna and EMC Engineer with the Airbus Defense and Space. His current research interests include computational electrodynamics in time domain, method of moments and fast algorithms for integral equations in frequency domain and computational electromagnetics applied to electromagnetic compatibility, antenna, and RADAR cross section.

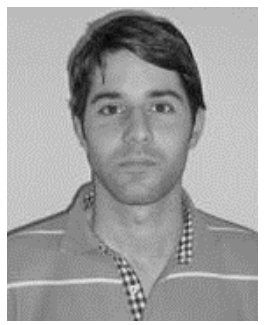

Miguel Ruiz Cabello was born in Granada, Spain. $\mathrm{He}$ received the B.Sc. and M.Sc. degrees in physics from the University of Granada, Granada, Spain, in 2008 and 2010, respectively. $\mathrm{He}$ is currently pursuing the Ph.D. degree with a specialization in HPC time-domain solvers for electromagnetic analysis and design.

Since 2010, he has been a Ph.D. Student in physics with the Department of Electromagnetism and Matter Physics. He has focused on the 7PM EU Project HIRF-SE as a Developer of the SEMBA-UGRFDTD solver.

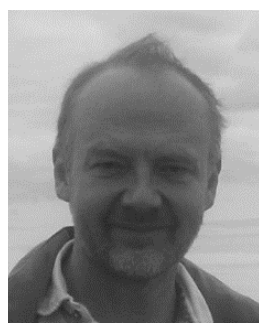

Martin P. Robinson (M'15) received the B.A. and M.A. degrees from the University of Cambridge, Cambridge, U.K., in 1986 and 1990, respectively, the M.Sc. degree in medical physics from the University of Aberdeen, Aberdeen, U.K., in 1990, and the Ph.D. degree in dielectric imaging from the University of Bristol, Bristol, U.K., in 1994.

He was with the UK's National Physical Laboratory for two years and for three years was with the Bristol Oncology Center, Bristol. He joined the University of York, York, U.K., in 1993. His current research interests include design for EMC, electromagnetic measurements, and the interaction of electromagnetic radiation with biological tissues.

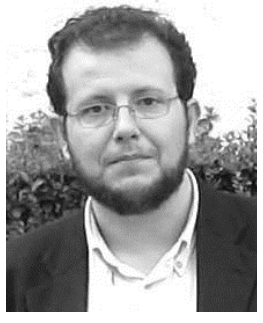

antenna design.
Salvador G. Garcia (M'93-SM'14) received the M.Sc. and Ph.D. degrees in physics from the University of Granada, Granada, Spain, in 1989 and 1994, respectively.

He is currently a full Professor with the Department of Electromagnetism and Matter Physics, University of Granada. He has authored over 80 refereed journal papers and book chapters and led several national and international projects. His current research interests include computational electromagnetics, electromagnetic compatibility, RCS, and Dr. Garcia was a recipient of the Extraordinary Award for his Ph.D. 